\title{
Edifenphos (Hinosan) reduces hyphal extension, hyphal growth unit length and phosphatidylcholine content of Fusarium graminearum A3/5, but has no effect on specific growth rate
}

\author{
Marilyn G. Wiebe, ${ }^{*}$ Geoffrey D. Robson and Anthony P. J. Trinci \\ Microbiology Group, Department of Cell and Structural Biology, School of Biological Sciences, Stopford Building, \\ University of Manchester, Manchester M13 9PT, UK
}

(Received 20 November 1989; revised 23 January 1990; accepted 12 February 1990)

\begin{abstract}
Low concentrations (41 $\mu \mathrm{M})$ of Hinosan (Edifenphos) inhibited hyphal extension and decreased the hyphal growth unit length and phosphatidylcholine content of Fusarium graminearum A3/5, but had no effect on specific growth rate. Thus, at low concentrations, Hinosan acted as a paramorphogen, increasing hyphal branching, but not inhibiting growth. The colony radial growth rate and specific growth rate of $F$. graminearum were significantly decreased at Hinosan concentrations of $82 \mu \mathrm{M}$ and $492 \mu \mathrm{M}$, respectively.
\end{abstract}

\section{Introduction}

Hinosan (Edifenphos; $O$-ethyl $S, S$-diphenylphosphorodithioate) was marketed by Bayer AG in 1968 to control Pyricularia oryzae on rice (Scheinpflug \& Jung, 1968). Maeda et al. (1970) reported that the related organophosphorus fungicide, Kitazin-P, inhibited incorporation of $\left[{ }^{14} \mathrm{C}\right]$ glucosamine into the cell wall of $P$. oryzae and they suggested that the fungicide inhibited chitin synthesis either directly, by inhibiting chitin synthase, or indirectly, by altering membrane permeability.

De Waard (1974) found that concentrations of Hinosan and Kitazin-P 5-10 times above their $\mathrm{ED}_{50}$ values for mycelial growth (about $100 \mu \mathrm{M}$ ) were required to inhibit incorporation of $\left[{ }^{14} \mathrm{C}\right] \mathrm{glucosamine}$ into the mycelium of $P$. oryzae $; \mathrm{ED}_{50}$ is the fungicide concentration which reduces fungal growth or enzyme activity by $50 \%$ compared with control values. The $\mathrm{ED}_{50}$ for inhibition of $P$. oryzae chitin synthase by Hinosan was also 10-fold above the $\mathrm{ED}_{50}$ for mycelial growth (Kodama et al., 1980). Craig \& Peberdy (1983a) and Leighton et al. (1981) reported that Kitazin-P inhibited the in vitro activity of chitin synthase from Aspergillus nidulans and Phycomyces sp. By contrast, Brillinger (1979) found that Coprinus cinereus chitin synthase was not inhibited by Kitazin-P, and De Waard (1974) reported that Pythium ultimum, an Oomycete fungus with

\footnotetext{
Abbreviations: PC, phosphatidylcholine; PE, phosphatidylethanol-
} amine; PI, phosphatidylinositol. walls containing cellulose but not chitin, was inhibited by both Hinosan and Kitazin-P. Thus, there are conflicting reports about the effects of organophosphorus fungicides on chitin synthesis.

Similarly, although Hinosan and Kitazin-P increased the membrane permeability of $P$. oryzae (De Waard, 1974), Kitazin-P had no effect on the membrane permeability of $A$. nidulans (Craig \& Peberdy, 1983a). There are also contradictory reports of the effects of Hinosan and Kitazin-P on phosphatidylcholine (PC) synthesis. For example, Akatsuka et al. (1971), Kodama et al. (1979) and Ross \& Brady (1985) reported that organophosphorus fungicides inhibited PC synthesis in fungi, but Craig \& Peberdy (1983b) found that Kitazin-P did not affect the PC content of $A$. nidulans. Uncertainty about the mode of action of organophosphorus fungicides is further increased by reports that Hinosan inhibited cutinase activity in Colletotrichum gloeosporioides (Dickman et al., 1983) and Fusarium solani (Sisler, 1986).

Steele \& Trinci (1975) showed that the mean rate of extension $(E)$ of hyphae in a fungal mycelium is a function of hyphal growth unit length ( $G$, the mean length of hypha associated with each hyphal tip) and specific growth rate $(\mu)$. Thus,

$$
E=G \mu
$$

Equation (1) predicts that, provided $\mu$ remains constant, $E$ will be directly related to $G$. Thus, factors which reduce $E$ without affecting $\mu$ will result in the formation of 
mycelia which branch more profusely than untreated mycelia.

The present paper describes the effects of various concentrations of Hinosan on the growth, branching and phospholipid composition of Fusarium graminearum and shows that at low concentrations the 'fungicide' acts as a paramorphogen (Tatum et al., 1949), inhibiting hyphal extension and increasing hyphal branching, but not altering specific growth rate.

\section{Methods}

Organism and media. Fusarium graminearum Schwabe A3/5 was obtained from Mr T. W. Naylor, Marlow Foods, Billingham, UK. The defined medium of Vogel (1956) was used with glucose $\left(10 \mathrm{~g} \mathrm{l}^{-1}\right)$ as the carbon source instead of sucrose. Vogel's mineral salts solution was prepared at $\times 50$ final concentration, sterilized by membrane $(0.2 \mu \mathrm{m}$ diameter) filtration and added to the glucose solution, which was sterilized by autoclaving at $121^{\circ} \mathrm{C}$ for $15 \mathrm{~min}$. Hinosan (provided by Imperial Chemical Industries, Jealott's Hill Research Station, UK) was dissolved in acetone (approximately 20\%, w/v, Hinosan/acetone) and mixed with sterile medium so that the fungicide was dispersed throughout the medium. Acetone was added to control media to give an equivalent final concentration $(0.5 \%, \mathrm{v} / \mathrm{v})$. For experiments in which medium was supplemented with choline (Sigma), a sterile solution of choline chloride $(100 \mathrm{~mm})$ was prepared by membrane filtration and this was added to the sterile glucose solution. For some experiments the medium was solidified with $15 \mathrm{~g}$ agar (Davis Gelatine; $15 \mathrm{~g} \mathrm{l}^{-1}$, final concentration).

Culture conditions. Liquid batch cultures of $F$. graminearum were grown in $20 \mathrm{ml}$ volumes of medium in $250 \mathrm{ml}$ Nephlos flasks (Trinci, 1972). Each flask was inoculated with $1 \mathrm{ml}$ of a suspension of macroconidia [final concentration about $5 \times 10^{4}$ spores (ml medium $)^{-1}$ ] in distilled water prepared by harvesting spores from 7- to 10-d-old cultures grown on agar-solidified Vogel's medium; the macroconidia harvested from these cultures were washed with $10 \mathrm{ml}$ of sterile distilled water. Cultures were incubated on a rotary shaker (with a throw of $2.5 \mathrm{~cm})$ at 200 r.p.m.

Germlings (a spore which has germinated to form a small, undifferentiated mycelium) of $F$. graminearum were grown in $9 \mathrm{~cm}$ diameter Petri dishes containing $20 \mathrm{ml}$ agar medium overlaid with Cellophane (PT 300, British Cellophane Ltd). Prior to use, the Cellophane was twice boiled for $15 \mathrm{~min}$ in distilled water to remove plasticizers. Cellophane-overlaid medium was inoculated with about 50 macroconidia, which were spread over the surface using a glass spreader; the Cellophane ensured that the mycelia grew in a single plane and could be photographed. Colonies of $F$. graminearum were grown in $9 \mathrm{~cm}$ diameter Petri dishes containing $20 \mathrm{ml}$ agar media which were inoculated centrally with $1.5 \mu \mathrm{l}$ of a suspension of macroconidia $\left(10^{6}\right.$ spores $\left.\mathrm{ml}^{-1}\right)$. All cultures were incubated at $25^{\circ} \mathrm{C}$.

Measurements of fungal growth and morphology. The specific growth rate $(\mu)$ and mean hyphal extension rate $(E)$ of mycelia cultured on agar medium were determined by time-lapse photomicroscopy as described by Trinci (1974). $E$ was calculated from:

$$
E=\frac{2\left(H_{\mathrm{t}}-H_{0}\right)}{B_{0}+B_{\mathrm{t}}}
$$

where $H_{0}=$ total hyphal length at zero time, $H_{\mathrm{t}}=$ total hyphal length $1 \mathrm{~h}$ later, $B_{0}=$ number of tips at zero time, and $B_{\mathrm{t}}=$ number of tips $1 \mathrm{~h}$ later. Measurements of hyphal growth unit lengths were made on mycelia which had been grown for 17-26 h on agar medium or for 20 $23 \mathrm{~h}$ in liquid medium. Mycelia with five or more hyphal tips were photographed at magnifications of $\times 40$ (control) or $\times 100$ (Hinosantreated cultures) and were then measured on the negatives at a magnification of $\times 10$ using a Shadowmaster (Baty \& Co.). Hyphal diameters were measured at a magnification of $\times 1000$ using an Olympus travelling micrometer eyepiece. Measurements of colony diameters were made with a rule at a magnification of $\times 10$ (Shadowmaster).

Phospholipid analysis. Lipids were extracted from fungal biomass following the procedure of Angus \& Lester (1972). The mycelium was grown at $25^{\circ} \mathrm{C}$ in $20 \mathrm{ml}$ liquid medium in $250 \mathrm{ml}$ Erlenmeyer flasks with or without $123 \mu \mathrm{M}$-Hinosan, or $100 \mu \mathrm{M}$-choline, on a rotary shaker at 200 r.p.m. Cultures were harvested in late exponential phase by filtration through two layers of muslin. Phospholipids were extracted three times for $15 \mathrm{~min}$ at $60^{\circ} \mathrm{C}$ in $10 \mathrm{ml}$ solvent $(95 \%$ aqueous ethanol $/ \mathrm{H}_{2} \mathrm{O} /$ diethyl ether/pyridine/concentrated ammonia, $15: 15: 5: 1: 0 \cdot 018$, by vol). The three extracts were combined and dried in a rotary evaporator at $45^{\circ} \mathrm{C}$. The lipid residue was redissolved in chloroform/methanol $(2: 1, \mathrm{v} / \mathrm{v})$, dried under a stream of nitrogen and stored at $-20^{\circ} \mathrm{C}$. The phospholipid extracts were dissolved in $0.05 \mathrm{ml}$ chloroform/methanol $(2: 1, v / v)$ and $100 \mu l$ was applied as a small spot to activated thin-layer chromatography plates (Merck, silica gel G, $20 \times 10 \mathrm{~cm})$. The plates were developed in two dimensions with the following solvents: (first direction) chloroform/methanol/ ammonia/ $\mathrm{H}_{2} \mathrm{O}(66: 27: 3: 0 \cdot 9$, by vol.), and (second direction) chloroform/methanol/acetic acid/ $\mathrm{H}_{2} \mathrm{O}(32: 10: 5: 1$, by vol.) for $20-30 \mathrm{~min}$, drying the plates between solvents to remove the ammonia. Phospholipids were visualized with a phosphomolybdate spray reagent (Dittmer \& Lester, 1964). The spots were marked and scraped into separate test tubes. Phosphate content was measured using the method of Rouser et al. (1970).

\section{Results}

Effect of Hinosan on the radial growth rate of colonies of F. graminearum $A 3 / 5$

Fig. 1 shows the effect of Hinosan on the radial growth rate $\left(K_{\mathrm{r}}\right)$ of colonies of $F$. graminearum A3/5. Untreated colonies had a $K_{\mathrm{r}}$ of $140 \pm 2 \mu \mathrm{m} \mathrm{h}^{-1}$ (mean \pm standard error). Hinosan concentrations of $4 \cdot 1$ and $8 \cdot 2 \mu \mathrm{M}$ caused slight but significant increases $(P<0.05)$ in $K_{\mathrm{r}}$, and significant decreases in $K_{\mathrm{r}}$ were observed at Hinosan concentrations between $82\left(K_{\mathrm{r}}=100 \pm 1 \mu \mathrm{m} \mathrm{h}^{-1}\right)$ and $820\left(K_{\mathrm{r}}=35 \pm 0 \cdot 5 \mu \mathrm{m} \mathrm{h}^{-1}\right) \mu \mathrm{M}$.

\section{Effect of Hinosan on the growth and branching of mycelia of F. graminearum A3/5 cultured on agar medium}

Inhibition (only about $65 \%$ of the spores germinated) of spore germination was only observed at $820 \mu \mathrm{M}$-Hinosan (results not shown). However, Hinosan concentrations of $246 \mu \mathrm{M}$ and below had no $(P>0.05)$ significant effect on the specific growth rate of $F$. graminearum A $3 / 5$, but growth of the fungus was decreased at higher fungicide concentrations (Table 1). By contrast, Hinosan concentrations as low as $41 \mu \mathrm{m}$ caused significant decreases 


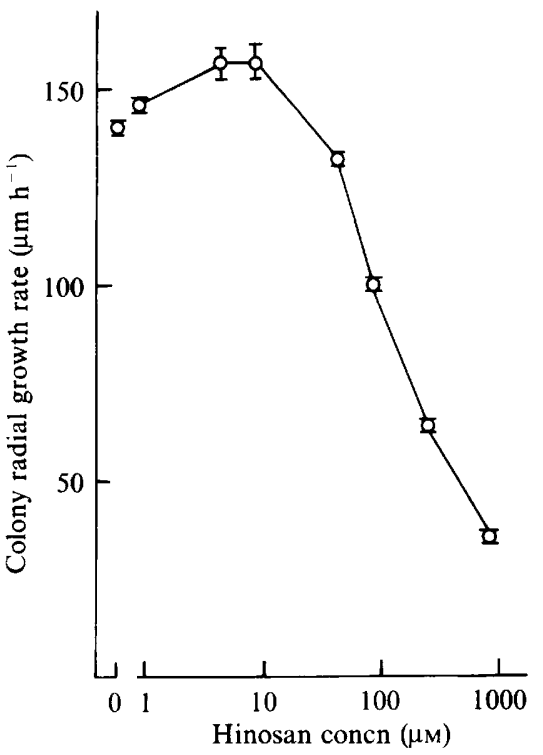

Fig. 1. Effects of Hinosan on the radial growth rate of $F$. graminearum A3/5. Cultures were grown on modified Vogel's agar medium at $25^{\circ} \mathrm{C}$. Error bars represent $95 \%$ confidence intervals (10 replicates per treatment).

Table 1. Effects of Hinosan on the specific growth rate, mean hyphal extension rate and hyphal growth unit length of mycelia of $F$. graminearum $A 3 / 5$ cultured at $25^{\circ} \mathrm{C}$ on Vogel's agar medium

The results are the means of 4-16(*), 22-29(†) and 26-100 (\$) replicates, respectively, and are shown \pm standard error. Figures in the same column with the same superscript letter $(a-e)$ are not significantly different $(P>0 \cdot 05)$.

\begin{tabular}{cccc}
\hline $\begin{array}{c}\text { Hinosan } \\
\text { concn } \\
(\mu \mathrm{M})\end{array}$ & $\begin{array}{c}\text { Specific growth } \\
\text { rate* }^{*}\left(\mu, \mathrm{h}^{-1}\right)\end{array}$ & $\begin{array}{c}\text { Mean hyphal } \\
\text { extension rate } \dagger \\
\left(E, \mu \mathrm{m} \text { per tip } \mathrm{h}^{-1}\right)\end{array}$ & $\begin{array}{c}\text { Hyphal growth } \\
\text { unit length } \\
(G, \mu \mathrm{m})\end{array}$ \\
\hline 0 & $0 \cdot 28 \pm 0 \cdot 01^{a}$ & $70 \pm 2^{a}$ & $266 \pm 7^{a}$ \\
41 & $0 \cdot 28 \pm 0 \cdot 01^{a}$ & $38 \pm 1^{b}$ & $141 \pm 3^{b}$ \\
82 & $0 \cdot 26 \pm 0 \cdot 01^{a}$ & $22 \pm 0 \cdot 6^{c}$ & $87 \pm 2^{c}$ \\
246 & $0 \cdot 26 \pm 0 \cdot 01^{a}$ & $15 \pm 0 \cdot 4^{d}$ & $62 \pm 1^{d}$ \\
492 & $0 \cdot 22 \pm 0 \cdot 01^{b}$ & $11 \pm 0 \cdot 6^{d e}$ & $48 \pm 1^{e}$ \\
820 & $0 \cdot 18 \pm 0 \cdot 01^{b}$ & $8 \pm 0 \cdot 4^{e}$ & $46 \pm 1^{e}$ \\
\hline \hline
\end{tabular}

$(P>0.05)$ in the mean hyphal extension rate and hyphal growth unit length of mycelia of $F$. graminearum A3/5.

\section{Effect of Hinosan on the branching of $F$. graminearum A3/5 in liquid medium}

Because L-sorbose affects the branching of mycelia of Neurospora crassa cultured on agar but not in liquid medium (Trinci \& Collinge, 1973), it cannot be assumed that the effects of Hinosan on hyphal extension and

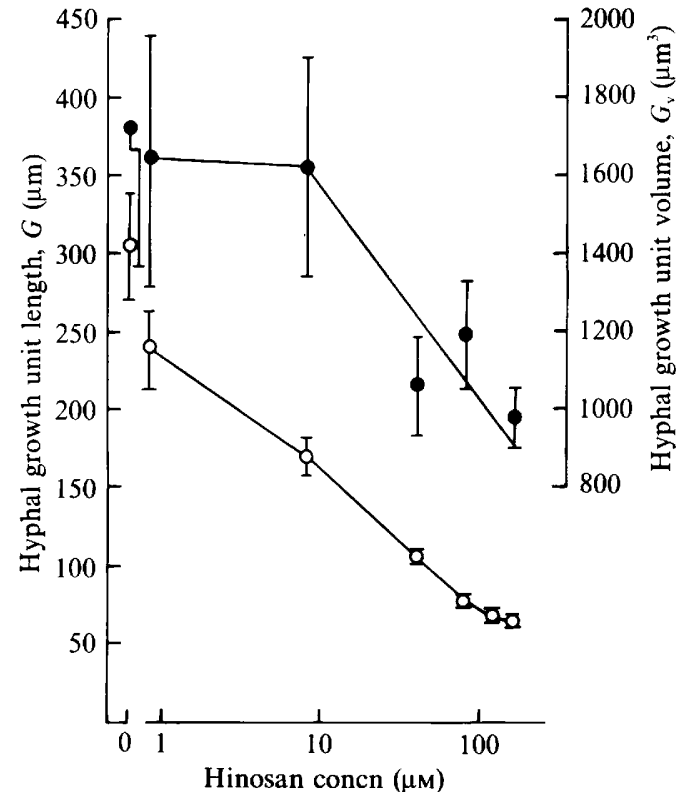

Fig. 2. Effects of Hinosan on the hyphal growth unit length $(G, O)$ and hyphal growth unit volume $\left(G_{\mathrm{v}}\right.$, calculated from equation 3,0$)$ of $F$. graminearum A3/5 cultured at $25^{\circ} \mathrm{C}$ on modified Vogel's liquid medium in shake flask cultures. Samples were taken $20-23 \mathrm{~h}$ after inoculation. Error bars represent $95 \%$ confidence intervals $(25$ replicates per treatment were made for $G$, and 100 diameters were measured per treatment to calculate $G_{v}$ ).

branching of mycelia of $F$. graminearum cultured on agar medium would also be observed in liquid culture. However, $F$. graminearum was more sensitive to Hinosan in liquid medium than on agar. Concentrations of Hinosan as low as $0.8 \mu \mathrm{M}$ caused a significant decrease $(P<0.05)$ in the hyphal growth unit length of mycelia of $F$. graminearum A3/5 cultured in shake flask, submerged culture (Fig. 2) and a $45 \%$ reduction in $G$ was observed at $8.2 \mu \mathrm{M}$ Hinosan; by contrast, on agar media, a $45 \%$ reduction in $G$ was only observed when the medium contained $41 \mu \mathrm{M}$ Hinosan (Table 1).

The hyphal growth unit volumes (Trinci, 1984) of mycelia of $F$. graminearum A3/5 cultured in shake flask culture were calculated from:

$$
G_{\mathrm{v}}=\pi r^{2} G
$$

where $G_{\mathrm{v}}=$ hyphal growth unit volume, $G=$ hyphal growth unit length, and $r=$ hyphal radius, assuming the hyphal filaments to be cylindrical. Hyphal growth unit volume decreased with increasing Hinosan concentration.

Phospholipid composition of $F$. graminearum A3/5 in the preseñce or absence of Hinosan

Kodama et al. (1980) showed that the primary mode of action of Hinosan in $P$. oryzae was to inhibit the 
Table 2. Phospholipid composition of F. graminearum A3/5 in the presence and absence of $123 \mu \mathrm{M}$-Hinosan and in $123 \mu \mathrm{M}$-Hinosan plus $100 \mu \mathrm{M}$-choline

Cultures grown at $25^{\circ} \mathrm{C}$ in shake flask cultures were harvested during late exponential phase and the phospholipids present in the biomass were extracted and measured as described in the Methods. Individual phospholipids are expressed as a percentage of the total phospholipid content of the biomass. Each analysis is the mean \pm standard error of nine replicates from two separate experiments. Figures in the same horizontal row with the same superscript letter are not significantly different $(P>0.05)$.

\begin{tabular}{lccc}
\hline \hline & \multicolumn{2}{c}{ F. graminearum A3/5 grown in the : } \\
\cline { 2 - 4 } & absence of & $\begin{array}{c}\text { presence of } \\
\text { Hinosan }\end{array}$ & $\begin{array}{c}\text { presence of } \\
\text { Hinosan and } \\
\text { choline }\end{array}$ \\
\hline Phospholipid & $48 \cdot 6 \pm 2 \cdot 7^{a}$ & $33 \cdot 4 \pm 1 \cdot 8^{b}$ & $31 \cdot 9 \pm 2 \cdot 9^{b}$ \\
Phosphatidylcholine (PC) & $19 \cdot 0 \pm 1 \cdot 7^{a}$ & $17 \cdot 0 \pm 1 \cdot 1^{a}$ & $16 \cdot 7 \pm 0 \cdot 8^{a}$ \\
Phosphatidylethanolamine (PE) & $7 \cdot 5 \pm 0 \cdot 6^{a}$ & $6 \cdot 6 \pm 0 \cdot 4^{a}$ & $7 \cdot 2 \pm 0 \cdot 5^{a}$ \\
Phosphatidylinositol (PI) & $5 \cdot 9 \pm 0 \cdot 4^{a}$ & $3 \cdot 9 \pm 0 \cdot 7^{a}$ & $4 \cdot 3 \pm 0 \cdot 4^{a}$ \\
Phosphatidylserine & $1 \cdot 5 \pm 0 \cdot 3^{a}$ & $4 \cdot 0 \pm 0 \cdot 7^{a}$ & $5 \cdot 7 \pm 1 \cdot 0^{a}$ \\
Phosphatidic acid & $2 \cdot 1 \pm 0 \cdot 2^{a}$ & $1 \cdot 6 \pm 0 \cdot 2^{a}$ & $1 \cdot 8 \pm 0 \cdot 2^{a}$ \\
Cardiolipin & $1 \cdot 0 \pm 0 \cdot 3^{a}$ & $3 \cdot 3 \pm 0 \cdot 4^{a}$ & $2 \cdot 5 \pm 0 \cdot 3^{a}$ \\
Phosphatidylglycerol & $0.9 \pm 0 \cdot 6^{a}$ & $1 \cdot 2 \pm 0 \cdot 4^{a}$ & $1 \cdot 8 \pm 0 \cdot 5^{a}$ \\
Unknown 1 & $\mathrm{ND}$ & $18 \cdot 2 \pm 2 \cdot 3^{a}$ & $21 \cdot 0 \pm 2 \cdot 4^{a}$ \\
Unknown 5 & & & \\
\hline \hline
\end{tabular}

ND, Not detectable.

methylation of $\mathrm{PE}$ to $\mathrm{PC}$. The PC level of $F$. graminearum A $3 / 5$ was reduced by $15 \%$ when $123 \mu \mathrm{M}$-Hinosan was included in the medium (Table 2) and there was a corresponding increase of $18 \%$ in the level of an unidentified phospholipid (unknown 5). No change in total phospholipid content was observed.

PC can be synthesized either by the methylation of PE (Bremer \& Greenberg, 1960) or from choline (Kennedy \& Weiss, 1956). Both Greenberg and Kennedy pathways have been observed in fungi, but the former (methylation) pathway appears to be dominant (Scarborough \& Nyc, 1967; Wilson \& Barran, 1983; Chin \& Bloch, 1988). If Hinosan inhibits only the methyltransferase and does not effect the Kennedy pathway, addition of exogenous choline to the medium should elevate the level of PC in Hinosan-treated cultures. Table 2 shows that PC levels were not significantly altered by addition of choline to the medium.

\section{Discussion}

Table 2 shows that $123 \mu \mathrm{M}$-Hinosan inhibited PC synthesis in $F$. graminearum. This result suggests that the mode of action of Hinosan in $F$. graminearum is similar to its mode of action in $P$. oryzae (Kodama et al., 1980), but differs from that in $A$. nidulans where no reduction in PC was observed (Craig \& Peberdy, 1983a). In P. oryzae,
Hinosan specifically inhibited the phospholipid $\mathrm{N}$ methyltransferase of the Greenberg pathway of choline synthesis. As the action of Hinosan appears to be the same as that of Kitazin-P, Hinosan probably also inhibits the incorporation of methyl groups into free choline (Yoshida et al., 1984).

In P. oryzae, Kitazin-P (50 p.p.m., $170 \mu \mathrm{M})$ did not inhibit incorporation of choline into PC (Kodama et al., 1979) and no inhibition was observed with low concentrations $(42 \mu \mathrm{M})$ of Hinosan (Kodama et al., 1980). It was concluded that Hinosan did not affect the Kennedy pathway for PC synthesis. Hinosan at 50 p.p.m. $(160 \mu \mathrm{M})$, however, did reduce choline incorporation by $P$. oryzae by almost $40 \%$ (Kodama et al., 1980). If the Kennedy pathway is not inhibited, then exogenous choline should be incorporated into PC, eliminating or reducing the effect of Hinosan on PC synthesis. However, Table 2 shows that PC levels in F. graminearum were not increased by addition of choline to the medium. Together with the result of Kodama et al. (1980), Table 2 suggests that Hinosan inhibits both pathways for PC synthesis, inhibition of the Kennedy pathway possibly being secondary to inhibition of the Greenberg pathway. However, it is also possible that the Kennedy pathway does not function in $F$. graminearum, although both pathways have been observed in Fusarium oxysporum (Wilson \& Barran, 1983). Addition of choline to the medium does not change the PC content of $F$. graminearum (Wiebe et al., 1989). 
The present results show the paramorphogenic effect of low concentrations of Hinosan on F. graminearum. For example, $41 \mu \mathrm{M}$-Hinosan had no effect on specific growth rate but caused significant decreases in hyphal extension rate and hyphal growth unit length (Table 1). Equation 1 predicts that any compound which causes a decrease in hyphal extension rate without affecting specific growth rate will inevitably have an effect on hyphal growth unit length. In Rhizoctonia cerealis, increased branching observed in the presence of validamycin A was correlated with a reduced PI content (Robson et al., 1989). Thus, the results of Robson et al. (1989), and the present results, suggest that the phospholipid composition of fungal membranes may influence fungal branching.

The interaction of membrane proteins with membrane lipids is well documented (Lee, 1987; Lenaz, 1987) and some fungal enzymes have specific phospholipid requirements for optimal activity (Duran \& Cabib, 1978; Vermeulen \& Wessels, 1983; Montgomery \& Gooday, 1985; Humphreys \& Gooday, 1984). Robson et al. (1989) suggested that if the activity $\left(K_{\mathrm{m}}, V\right)$ of enzymes involved in tip extension is diminished when the phospholipid environment is altered, then the mean hyphal extension rate will be reduced. If, in addition, specific growth rate is not affected by the change in phospholipid composition, the reduced hyphal extension rate will lead to an increase in branching (equation 1; Trinci, 1984). Results presented in this paper suggest that Hinosan may be acting in this manner in $F$. graminearum and that the reduced PC content of the membrane may affect the activity of chitin synthase.

We thank the Natural Science and Engineering Research Council of Canada and Marlow Foods for supporting this research.

\section{References}

Akatsuka, T., Kodama, O. \& Yameda, H. (1977). A novel mode of action of kitazin $\mathbf{P}$ in Pyricularia oryzae. Agricultural and Biological Chemistry 41, 2111-2112.

ANGUS, W. W. \& Lester, R. L. (1972). Turnover of inositol and phosphorus containing lipids in Saccharomyces cerevisiae ; extracellular accumulation of glycerophosphatidylinositol derived from phosphatidylinositol. Archives of Biochemistry and Biophysics 151, 483495 .

BREMER, J. \& GREENBERG, D. M. (1961). Methyl transferring enzyme system of microsomes in the biosynthesis of lecithin (phosphatidylcholine). Biochimica et Biophysica Acta 46, 205-216.

BRILLINGER, G. U. (1979). Metabolic products of microorganisms. 181. Chitin synthase from fungi, a test model for substances with insecticidal properties. Archives of Microbiology 121, 71-74.

CHIN, J. \& BLOCH, K. (1988). Phosphatidylcholine synthesis in yeast. Journal of Lipid Research 29, 9-14.

Craig, G. D. \& Peberdy, J. F. (1983a). The mode of action of $S$-benzyl $O, O$-di-isopropyl phosphorothioate and dichloran on Aspergillus nidulans. Pesticide Science 14, 17-24.
Craig, G. D. \& Peberdy, J. F. (1983b). The effect of $S$-benzyl $O, O$-diisopropylphosphorothioate (IBP) and dichloran on the total lipid, sterol and phospholipids in Aspergillus nidulans. FEMS Microbiology Letters 18, 11-14

DE WAARD, M. A. (1974). Mechanisms of action of the organophosphorus fungicide Pyrazophos. PhD thesis. Mededelingen Landbouwhogeschool Wageningen. Wageningen: H. Veenman \& Zonen, B. V.

Dickman, M. B., Patil, S. S. \& Kolattudy, P. E. (1983). Effects of organophosphorus pesticides on cutinase activity and infection of papayas by Colletotrichum gloeosporioides. Phytopathology 73, $1209-1214$.

DitTMER, J. C. \& LeSTER, R. L. (1964). A simple specific spray reagent for the detection of phospholipids on thin-layer chromatograms. Journal of Lipid Research 5, 126-127.

DuRAN, A. \& CABIB, E. (1978). Solubilization and partial purification of yeast chitin synthetase. Journal of Biological Chemistry 253, 4419-4425.

Humphreys, A. M. \& GoOday, G. W. (1984). Phospholipid requirement of microsomal chitinase from Mucor mucedo. Current Microbiology 11, 187-190.

KenNedy, E. P. \& Weiss, S. B. (1956). The function of cytidine coenzymes in the biosynthesis of phospholipids. Journal of Biological Chemistry 222, 193-214.

Kodama, O., Yamada, H. \& Axatsuxa, J. (1979). Kitazin P, inhibitor of phosphatidylcholine biosynthesis in Pyricularia oryzae. Agricultural and Biological Chemistry 43, 1719-1725.

Kodama, O., Yamashita, K. \& AKatsuka, J. (1980). Edifenphos, inhibitor of phosphatidylcholine biosynthesis. in Pyricularia oryzae. Agricultural and Biological Chemistry 44, 1015-1021.

LEE, A. G. (1987). Interactions of lipids and proteins: some general principals. Journal of Bioenergetics and Biomembranes 19, 581-603.

Leighton, T., Marks, E. \& Leighton, F. (1981). Pesticides: insecticides and fungicides are chitin synthesis inhibitors. Science 213, 905-907.

LENAZ, G. (1987). Lipid fluidity and membrane protein dynamics. Bioscience Reports 7, 823-837.

Maeda, T., Abe, H., Kakiki, K. \& Misato, T. (1970). Studies on the mode of action of organophosphorus fungicide, Kitazin. Part II. Accumulation of an amino sugar derivative from kitazin-treated mycelia of Pyricularia oryzae. Agricultural and Biological Chemistry 34, 700-709.

Montgomery, G. W. G. \& Gooday, G. W. (1985). Phospholipidenzyme interactions of chitin synthase of Coprinus cinereus. FEMS Microbiology Letters 27, 29-33.

Robson, G. D., Kuhn, P. L. \& Trinci, A. P. J. (1989). Effect of validamycin A on the inositol content and branching of Rhizoctonia cerealis and other fungi. Journal of General Microbiology 135, 739-750.

Ross, D. C. \& BRADY, U. E. (1985). Organophosphate pesticides and DMSO affect mycelial growth and phosphatidylcholine biosynthesis in an entomopathic fungus. Journal of Entomological Science 20 $287-293$.

Rouser, G., Fleischer, S. \& Yamamoto, A. (1970). Two dimensional thin layer chromatographic separation of polar lipids and determination of phospholipids by phosphorus analysis of spots. Lipids 5,494 496.

SCARborough, G. A. \& Nyc, J. F. (1967). Methylation of ethanolamine phosphatides by microsomes from normal and mutant strains of Neurospora crassa. Journal of Biological Chemistry 242, 238-242.

ScheinPflug, H. \& JUNG, H. F. (1968). Organophosphates for the control of fungal diseases of crops. Pflanzenschutz-Nachrichten 21, $79-91$

Steele, G. C. \& Trinci, A. P. J. (1975). Morphology and growth kinetics of differentiated and undifferentiated mycelia of Neurospora crassa. Journal of General Microbiology 91, 362-368.

SISLER, H. D. (1986). Control of fungal diseases by compounds acting as antipenetrants. Crop Protection 5, 306-313.

Tatum, E. L., Barrat, R. W. \& Cutter, V. M. (1949). Chemical induction of colonial paramorphs in Neurospora and Syncephalastrum. Science 109, 509-511.

TrINCI, A. P. J. (1972). Culture turbidity as a parameter of mould growth. Transactions of the British Mycological Society 58, 467-473. 
Trincl, A. P. J. (1974). A study of the kinetics of hyphal extension and branch initiation of fungal mycelia. Journal of General Microbiology 81, 225-236.

TRINCI, A. P. J. (1984). Regulation of hyphal branching and hyphal orientation. In Ecology and Physiology of the Fungal Mycelium (Eighth Symposium of the British Mycological Society), pp. 23-52. Edited by D. H. Jennings \& A. D. M. Rayner. Cambridge: Cambridge University Press.

Trinci, A. P. J. \& Collinge, A. J. (1973). Influence of L-sorbose on growth and morphology of Neurospora crassa. Journal of General Microbiology 78, 179-192.

Vermuelen, C. A. \& Wessels, J. G. H. (1983). Evidence for a phospholipid requirement of chitin synthase in Schizophyllum commune. Current Microbiology 8, 67-71.
VoGEL, H. J. (1956). A convenient growth medium for Neurospora (Medium N). Microbial Genetics Bulletin 13, 42-44.

Wiebe, M. G., Robson, G. D. \& Trinci, A. P. J. (1989). Effect of choline on the morphology, growth and phospholipid composition of Fusarium graminearum. Journal of General Microbiology 135, $2155-2162$.

Wilson, A. C. \& Barran, L. R. (1983). Effect of temperature on phosphatidylcholine synthesis by hyphae of Fusarium oxysporum f.sp. lycopersici. Transactions of the British Mycological Society 80, $113-116$.

Yoshida, M., Moriya, S. \& Uesugi, Y. (1984). Observations of transmethylation from methionine into choline in the intact mycelia of Pyricularia oryzae by ${ }^{13} \mathrm{C}$ NMR under the influence of fungicides. Journal of Pesticide Science 9, 703-708. 